\title{
Comparison of the prevalence and characteristics of inpatient adverse events using medical records review and incident reporting
}

\author{
W M Macharia, ${ }^{1}$ MB ChB, MMed (Paeds), MSc; C M Muteshi, ${ }^{2}$ MB ChB, MMed (O\&G); S Z Wanyonyi, ${ }^{2}$ MB ChB, MMed (O\&G); \\ A M Mukaindo, ${ }^{2}$ MMed (O\&G); A Ismail, ${ }^{1} \mathrm{MB}$ ChB, MMed (Paeds); H Ekea, ${ }^{3}$ MB ChB; A Abdallah, ${ }^{4}$ MB ChB, MMed (Surg); \\ J M Tole, ${ }^{1}$ MB ChB, MMed (Paeds); A K Ngugi, ${ }^{5}$ BSc, MSc, PhD \\ ${ }^{1}$ Department of Paediatrics, Faculty of Health Sciences, Aga Khan University, Nairobi, Kenya \\ ${ }^{2}$ Department of Obstetrics and Gynaecology, Faculty of Health Sciences, Aga Khan University, Nairobi, Kenya \\ ${ }^{3}$ Department of Medicine, Faculty of Health Sciences, Aga Khan University, Nairobi, Kenya \\ ${ }^{4}$ Department of Surgery, Faculty of Health Sciences, Aga Khan University, Nairobi, Kenya \\ ${ }^{5}$ Population Health Sciences, Faculty of Health Sciences, Aga Khan University, Nairobi, Kenya
}

Corresponding author: W M Macharia (william.macharia@aku.edu)

Background. Information on adverse events (AEs) in hospitalised patients in developing countries is scanty.

Objective. To compare the magnitude and characteristics of inpatient AEs in a tertiary, not-for-profit healthcare facility in Kenya, using medical records review and incident reporting.

Methods. Estimation of prevalence was done using incidents reported in 2010 from a random sample of medical records for hospital admissions. Nurse reviewers used 18 screening criteria, followed by physician reviewers to confirm occurrence. An AE was defined as an unexpected clinical event (UE) associated with death, disability or prolonged hospitalisation not explained by the disease condition. The kappa statistic was used to estimate inter-rater agreement, and analysis was done using logistic regression.

Results. The study identified 53 UEs from 2000 randomly selected medical records and 33 reported UEs from 23026 admissions in the index year. The prevalences of AEs from medical records review and incident reports were $1.4 \%$ (95\% confidence interval (CI) $0.9-2.0)$ and $0.03 \%$ (95\% CI 0.012 - 0.063), respectively. Compared with incident reporting, review of medical records identified more disability ( $13.2 \%$ v. $0 \% ; p=0.03)$ and prolonged hospital stays $(43.4 \%$ v. $18.2 \% ; p=0.02)$.

Conclusions. Review of medical records is preferable to incident reporting in determining the prevalence of AEs in health facilities with limited inpatient quality improvement experience. Further research is needed to determine whether staff education and a positive culture change through promotion of non-punitive UE reporting or a combination of approaches would improve the comprehensiveness of $\mathrm{AE}$ reporting.

S Afr Med J 2016;106(10):1021-1036. DOI:10.7196/SAMJ.2016.v106i10.10619

The incidence of medical adverse events (AEs) in hospitalised patients was estimated at $9.2 \%$ (interquartile range 4.6 - 12.4) by de Vries et al. ${ }^{[1]}$ in 2008 . An event was defined as an incident resulting in death, a prolonged hospital stay or disability but not attributable to the underlying medical condition. The Canadian AEs study ${ }^{[2]}$ found a rate of $7.5 \%$ from review of 3745 medical records in four hospitals. Public hospitals in a multicentre study conducted in Africa and the Middle East had a range of $2.5-18.4 \%{ }^{[3]}$ Other methods used to assess AEs include incident reports, interviewing of healthcare providers, direct observation, external audits, confidential inquiries and complaints. Use of a hybrid electronic medical record system has demonstrated some benefit in combining prospective and retrospective approaches to uncover surgical AEs. Laing et al. ${ }^{[4]}$ identified $71.4 \%$ errors prospectively and $28.6 \%$ retrospectively. They found that a tick-box system improved the quality of documentation, which could help solve the disadvantage inherent in retrospective reviews. ${ }^{[5]}$ Prospective approaches involve voluntary written documentation or reporting of incidents, with concerns of underreporting when a culture of quality monitoring is not entrenched. ${ }^{[6]}$

\section{Objective}

The practice of AE reporting has not gained ground in many institutions in low-income countries. This may be due to inadequate awareness of its importance in informing service quality improvement to minimise harm to patients. This study was carried out with the objective of comparing the prevalence and patterns of AEs in hospitalised patients, using review of medical records and incident reporting.

\section{Methods}

The study was carried out in a 254-bed tertiary hospital in Nairobi, Kenya. The hospital has $\sim 20000$ admissions per annum, comprising medical, surgical, paediatric and obstetric patients. A retrospective review of randomly selected medical records of patients admitted in 2010 was undertaken to identify the presence or absence of AEs documented in the index year. All inpatient medical records and reported clinical incidents for the index year were eligible for inclusion. When more than one incident occurred in a patient in the index year, all were included as separate occurrences provided they were not related.

Using an estimated AE prevalence of $14.5 \%,{ }^{[3]}$ power of $80 \%$ and $5 \%$ precision, a sample size estimate of about 200 medical records was determined adequate. We assumed that $20 \%$ of the records would be incomplete and therefore added an extra 40 medical charts, giving a total sample size of 245 records. However, in order to obtain a larger number to enable meaningful comparison, we 
opted to increase the number of randomly sampled files to 2000 . All incidents recorded in the year were reviewed for fulfillment of predefined criteria for unintended or adverse clinical events. All the 1665 incidents reported in the index year were included in the review.

Unexpected clinical outcomes were defined as unexpected clinical events (UEs) to distinguish them from AEs. Events had to have: (i) occurred at any time before the index admission and been detected during, or been responsible for, the index admission; (ii) occurred and been detected during the index admission; or (iii) occurred during the index admission but been detected on a subsequent admission. An AE was defined as an incident that resulted in death, disability or a prolonged hospital stay and was not explainable by an underlying medical condition. Other occurrences were defined as UEs.

Two experienced nurses used RF1 forms (Appendix 1) to screen for unexpected events using 18 criteria comprising conditions or circumstances commonly associated with AEs. ${ }^{[2,3]}$ Four physicians then reviewed selected files using RF2 forms (Appendix 2). A panel of content experts in the surgical and medical fields was identified to act as a resource for technical input, but not to determine the occurrence of incidents. All reviewers underwent 2-day training. Pilot testing was conducted using a convenience sample of 20 records to ensure comprehension of the study techniques.

The nurse reviewers ensured that the physicians were not assigned to review medical records of patients in whose care they could previously have participated.

The physician reviewers then scrutinised all the records that screened positive using RF2 forms for the presence of AEs. They then categorised incidents according to severity, location where the event occurred, attribution and preventability.

Incident reports were identified by accessing both computerised records and paper files to determine the number of events reported in the year, the nature of the incidents and the circumstances surrounding them. Initial screening was done to exclude non-clinical incidents. Information on remedial measures taken following rootcause analysis was abstracted and entered into data collection forms. A random sample of $10 \%$ of the medical records was subjected to a second review by a reviewer with longer experience in similar studies, and who was not involved in the earlier reviews, for the purpose of validation.

The primary outcome measure was the prevalence of AEs, while secondary outcomes included UEs, circumstances leading to the UEs, site of incident occurrence and preventability.

The prevalence of AEs from medical records review was computed as a percentage using the total number of AEs as numerator and the total number of sampled admissions as denominator. Similarly, for incident reporting the numerator was the number of AEs and the denominator the total number of hospital admissions in the index year. A $p$-value of $<0.05$ was considered significant, and 95\% confidence intervals (CIs) were determined around primary outcome estimates. The $\chi^{2}$ test was used to compare independent categorical variables. Prevalence and bias-adjusted kappa statistics were used to estimate inter-rater agreement, with a value of $<0.4$ considered poor agreement, $0.4-0.6$ moderate agreement and $>0.6$ good agreement. ${ }^{[12]}$

The study was approved by the World Health Organization (Ref: PS09004) and the Aga Khan University Hospital Ethics Review Committees (AKU/REC-06052011). Anonymous record keeping was used to delink case records from study data, and patient confidentiality was maintained.

\section{Results}

There were 23026 hospital admissions in the year 2010, from which 2000 records were randomly selected for review. Of these, 317 were screening criteria-positive, with 53 (2.7\%; 95\% CI 2.0 3.5) having documented occurrence of a UE, but only $28 / 2000$ cases were associated with disabilities, a prolonged hospital stay or disabilities not attributable to the primary medical conditions, giving a prevalence of $1.4 \%$ (95\% CI $0.9-2.0)$. During the same study period, 233/1 $665(14.0 \%)$ of the reported incidents were of a clinical nature. From the total of 23026 admissions for 2010, 33 (0.14\%, 95\% CI $0.10-0.20$ ) had UEs but only 7 fulfilled the criteria for an AE, giving a prevalence of $0.03 \%$ ( $95 \%$ CI $0.01-0.06$ ).

Forty-three out of $428(10.0 \%)$ of the combined medical review and incident reports were reviewed by the 'expert reviewer' for validation. The inter-reviewer agreement for the reviewers was moderate at $\kappa=0.40(95 \%$ CI $0.13-0.66)$. The figure improved marginally to $\kappa=0.45$ (95\% CI 0.38 - 0.74) after adjusting for prevalence and bias.

Evidence of healthcare causation was identified in $31 / 53$ cases (58.5\%) from medical records and in 20/33 cases $(60.6 \%)$ by incident reporting. Comparing review of medical records with incident reporting, disability $(13.2 \%$ v. $0 \%)$ and prolonged hospital stay $(43.4 \%$ v. $18.8 \%)$ were more likely to be detected from review of records (Table 1).

The majority of UEs took place in the study hospital, predominantly in the patient's ward or room (Table 2).

The most frequently observed consequence of UEs from both data sources was prolonged hospital stay. Of the UEs detected by medical review, 11/15 (73.3\%) occurred while the patient was hospitalised and 4/15 (26.7\%) outside the facility.

Thirty-one of 53 medical records (58.5\%) attributed UE causation to healthcare management. Another $77.4 \%$ (95\% CI 52.0 - 87.8) of the events were non-procedure-related. Drugs were associated with UEs in $86.9 \%$ and $80.0 \%$ according to medical records and incident reports, respectively. Other health products that contributed to UEs, but less commonly, were blood products, medical devices and medical equipment. Clinical circumstances associated with UEs as determined by medical records and incident reports are shown in Table 3.

Of procedure-related UEs, $87.1 \%$ (95\% CI 70.2 - 96.4) occurred during therapeutic interventions. Medical records provided better prediction of UEs than incident reporting (62.3\% v. $36.4 \%$; $p=0.02)$.

Table 1. Nature of UEs identified from the medical records and incident report reviews

\begin{tabular}{|c|c|c|c|}
\hline Description of AE & $\begin{array}{l}\text { Medical record } \\
(N=53), n(\%)\end{array}$ & $\begin{array}{l}\text { Incident report } \\
(N=33), n(\%)\end{array}$ & $p$-value \\
\hline \multicolumn{4}{|c|}{ Events associated with death } \\
\hline Yes & $0(0.0)$ & $1(3.0)$ & 0.38 \\
\hline No & $53(100.0)$ & $32(97.0)$ & \\
\hline \multicolumn{4}{|c|}{ Events associated with disability } \\
\hline Yes & $7(13.2)$ & $0(0.0)$ & 0.03 \\
\hline No & $46(86.8)$ & $33(100.0)$ & \\
\hline \multicolumn{4}{|c|}{ Events associated with prolonged stay } \\
\hline Yes & $23(43.4)$ & $6(18.2)$ & \\
\hline No & $30(56.6)$ & $27(81.8)$ & 0.02 \\
\hline \multicolumn{4}{|c|}{ Healthcare causation/preventability } \\
\hline Yes & $31(58.5)$ & $20(60.6)$ & 0.85 \\
\hline No & $22(41.5)$ & $13(39.4)$ & \\
\hline
\end{tabular}


Table 2. Location of the occurrence of UEs as identified by the medical records and incident report reviews

\begin{tabular}{llll}
\hline & $\begin{array}{l}\text { Medical } \\
\text { record, } \boldsymbol{n} \text { (\%) }\end{array}$ & $\begin{array}{l}\text { Incident } \\
\text { report, } \boldsymbol{n} \text { (\%) }\end{array}$ & $\boldsymbol{p}$-value \\
\hline Location of occurrence & & & \\
Outside study hospital & $9(29.0)$ & $2(10.0)$ & 0.1 \\
Inside study hospital & $22(71.0)$ & $18(90.0)$ & \\
Total & 31 & 20 & \\
If inside, clinical unit of admission & & 0.16 \\
Yes & $19(86.4)$ & $18(100.0)$ & \\
No & $3(13.6)$ & 0 & \\
Total & 22 & 18 & \\
Exact location of occurrence inside hospital & \\
Theatre/ICU & $2(9.1)$ & $4(22.2)$ & \\
Outpatient clinic/ & $9(40.9)$ & $3(16.7)$ \\
A\&E/others & & & \\
Patient's room & $7(31.8)$ & $8(44.4)$ & \\
Labour and delivery & $4(18.2)$ & $3(1.7)$ \\
Total & 22 & 18
\end{tabular}

Table 3. Clinical circumstances associated with UEs as determined by medical records and incident report reviews

\begin{tabular}{|c|c|c|c|}
\hline & $\begin{array}{l}\text { Medical } \\
\text { record, } n(\%)\end{array}$ & $\begin{array}{l}\text { Incident } \\
\text { report, } n(\%)\end{array}$ & $p$-value \\
\hline \multicolumn{4}{|l|}{ Type of management } \\
\hline Prevention/diagnosis & $4(12.9)$ & $1(5.0)$ & 0.64 \\
\hline Therapeutic & $27(87.1)$ & $19(95.0)$ & \\
\hline Total & 31 & 20 & \\
\hline \multicolumn{4}{|l|}{ Cause of UE } \\
\hline Delay in implementation & $3(9.7)$ & $1(5.3)$ & 0.84 \\
\hline Error in implementation & $7(22.6)$ & $5(26.3)$ & \\
\hline $\begin{array}{l}\text { Other unavoidable/ } \\
\text { unidentified }\end{array}$ & $21(67.7)$ & $13(68.4)$ & \\
\hline Total & 31 & 19 & \\
\hline \multicolumn{4}{|l|}{ Procedure-related UE } \\
\hline Yes & $7(22.6)$ & $7(36.8)$ & 0.34 \\
\hline No & $24(77.4)$ & $12(63.2)$ & \\
\hline Total & 31 & 19 & \\
\hline \multicolumn{4}{|c|}{ Nature of procedure-related UE } \\
\hline Surgery/endoscopy & $4(57.1)$ & $1(14.3)$ & 0.27 \\
\hline Minor/instrumental & $3(42.9)$ & $6(85.7)$ & \\
\hline Total & 7 & 7 & \\
\hline \multicolumn{4}{|c|}{ Patient's global health-related UE } \\
\hline Yes & $17(54.8)$ & $6(30.0)$ & 0.1 \\
\hline No & $14(55.2)$ & $14(70.0)$ & \\
\hline Total & 31 & 20 & \\
\hline
\end{tabular}

However, the two methods did not differ in ability to detect preventability of UEs (30.2\% v. $42.4 \%$; $p=0.18$ ).
According to medical records, the underlying health status of the patient alone could have contributed to the occurrence of UEs in $64.7 \%$ of patients (95\% CI 46.5 - 80.3). Eight patients experienced UEs that were not associated with prolonged hospital stay, disability or death so were considered not to be serious, in keeping with the a priori definition. Incident reports identified 5 cases related to a therapeutic intervention, in 2 of which there was an error during administration, while the rest were considered unavoidable or due to undeterminable circumstances. Three cases were rather complex to manage, so it was not possible to attribute their cause to any specific care omission or commission.

Of 19 cases categorised by degree of urgency, 4 were of moderate urgency while 15 were of low or no urgency. Four of 6 events were related to inadequate communication or reporting by the healthcare team. Seventy-five percent of cases (9/12) could have benefited to a great extent or moderately if appropriate management had been given. The risk that a UE or AE would have resulted from management provided was moderate to high in $57.9 \%$ of the patients with UEs (95\% CI 33.5 - 79.7\%). Reviewers considered that $81 \%$ of competent healthcare professionals would have managed the patients with UEs in a similar manner.

\section{Discussion}

Our previous work in a multicentre study involving review of medical records in public hospitals in Africa and the Middle East found a prevalence of AEs of $2.5-18.4 \%{ }^{[3]}$ The mean in the two hospitals in Kenya was $14.5 \%$, which is much higher than the $1.4 \%$ in the medical records review in this study. A similar methodology was used for the medical records review, and some reviewers who had participated in the previous study were involved. This difference may be attributed to the higher standards of care in the current study site, which is a tertiary not-for-profit academic medical centre that at the time of the study was in the preparatory stages of Joint Commission International accreditation, which was eventually granted in 2013. Whereas AEs that are not associated with serious complications could fail to be documented, leading to underestimation, we only sought to document the severe ones that would readily be picked up from records, since death, disabilities and prolonged hospital stay would be evident.

Review of medical records in this study identified many more AEs than incident reporting ( $1.4 \%$ v. $0.03 \%)$, clearly indicating that many important events are never reported. This very low reporting may be a result of fear of being held responsible for omissions or commissions. It could also be due to inadequate staff education on the importance of reporting.

The majority of AEs in this study occurred in the rooms in which patients were receiving medical care. There can be major variations in the quality of care provided to hospitalised patients at different levels of care, especially for trauma patients. ${ }^{[7]}$ Medical records review was better than incident reporting at identifying events that resulted in disability $(13.2 \%$ v. $0 \% ; p=0.03)$ and prolonged hospitalisation $(43.4 \%$ v. $18.2 \%$; $p=0.02)$. This is not really surprising considering that these factors were triggers for AE scrutiny in medical records reviews, while incident reporting is expected to be spontaneous. An important drawback of the medical records approach is inability to fully reveal circumstances surrounding the event. Complementing review of records with structured morbidity and mortality meetings may be valuable. Clarke et al.$^{[8]}$ demonstrated the usefulness of such audit meetings in dissecting out human error contributions. Incident reporting requires a change in institutional culture so that it is not punitive to those who disclose UEs. Where such a culture is not entrenched, 
non-reporting would be even higher for patients on treatment for chronic and complex conditions, as true AEs in such patients may erroneously be assumed to be inevitable. There is also a tendency for health professionals to interpret AEs as expected complications of medical procedures even when they are not, and therefore failing to report them. This problem can, however, be overcome through comprehensive medical record review. ${ }^{[9]}$

Christians-Dingelhoff et al. ${ }^{[10]}$ found that only $3.6 \%$ of AEs identified by record review were identified by other reporting methods. As in our study, others also found different methods to be complementary with little overlap in reporting of AEs. ${ }^{[11]}$ This is especially important because some AEs not captured by incident reports would not have been detected using alternative methods, thus concealing opportunities for service improvement. Complementing incident reports with medical records review and other reporting modalities would ensure a more comprehensive assessment of AEs. Clarke et al. ${ }^{[12]}$ demonstrated how modern error theory used in commercial aviation could be exploited in health to identify missed injuries in trauma. However, we did not find any literature on how this could be applied for missed AEs in facility incidence or prevalence estimation.

By its nature, incident reporting included a large number of nonclinical reports that tended to mask non-reporting of important clinical incidents. A good reporting system should be able to clearly separate clinical and non-clinical incidents, as users are different. Our review was made possible by robust medical record keeping and an electronic incident reporting system. Lack of appropriate systems for data retrieval and analysis would be a major constraint for resource-deprived facilities. Nurse and physician reviewers with ample experience and training, as we had in our study, help to minimise observer variability.

Management flaws were responsible for nearly $60 \%$ of the AEs, with management itself likely to have contributed to AEs in $58 \%$ of cases. Irrespective of the data collection method used, $>80 \%$ of AEs related to medications. Our reviewers estimated that in $75 \%$ of AEs the patient could have benefited from more appropriate care than had been provided.

Level of agreement among physicians completing RF2 forms was estimated using the prevalence-adjusted kappa, as described by Bennet $e t$ al. in 1954 and elaborated by Nam, ${ }^{[13]}$ to give a more reliable degree of agreement. This reflected an acceptable chance-corrected agreement of 0.45 , representing moderately good agreement.

We observed that review of medical records also exposed more disabilities and prolonged hospital stay associated with the events. Laing et al., ${ }^{[4]}$ using a hybrid electronic method, found almost three times more events prospectively than by scrutiny of records. Although the settings may not be comparable, given that the studies were carried out in different continents serving different patient populations, the difference in findings suggests major under-reporting of incidents in our study. Institutional cultural change through staff education on the purpose of reporting and assurance that reporting will not result in punishment may be needed to alter perceptions and practice. There is little overlap in the events, emphasising the need for combining different approaches to be comprehensive. The search for innovative approaches to identify the many factors that impact on quality of care to hospitalised patients continues, even as known combined approaches demonstrate promise.

\section{Conclusions}

Review of medical records is preferable to incident reporting in health facilities with limited inpatient quality improvement experience. The approach identifies more AEs and exposes more factors associated with events. Further research is needed to determine whether staff education and positive culture change through promotion of nonpunitive reporting, or a combination of approaches, would improve comprehensiveness of AEs reporting.

Authors' contributions. WMM was involved in conceptualisation, proposal writing, analysis and drafting of the final manuscript. CMM participated in study implementation, analysis and drafting of the final manuscript. SZW was involved in conceptualisation, proposal writing and drafting of the final manuscript. AMM, AI, HE, AA and JMT all participated in data collection and drafting of this manuscript. AKN participated in data analysis and drafting of the manuscript. All the authors reviewed the manuscript and approved submission.

Acknowledgements. This work was supported by the World Health Organization Small Grants Program (WHO Ref: 2011/167789-0). We wish to acknowledge the enormous amount of guidance we received from Nittita Prasopa-Plaizier (WHO, Geneva) during the proposal writing and manuscript preparation stages. Georghe Banica (WHO, Geneva) similarly provided us with requisite administrative support for completion of this work. Janet Musia made invaluable input in data and project management.

1. De Vries EN, Ramrathan MA, Smorenburg SM, Gouma DJ, Boermeester MA. The incidence and nature of in-hospital adverse events: A systematic review. Qual Saf Health Care 2008;17(3):216-223. DOI:10.1136/qshc.2007.02362

2. Baker GR, Norton PG, Flintoft V, et al. The Canadian adverse events study: The incidence of adverse events among hospital patients in Canada. CMAJ 2004;170(11):1678-1686. DOI:10.1503/ cmaj.1040498.

3. Wilson RM, Michel P, Olsen S, et al. Patient safety in developing countries: Retrospective estimation of scale and nature of harm to patients in hospital. BMJ 2012;344:e832. DOI:10.1136/bmje.e832

4. Laing G, Bruce J, Skinner D, et al. Using a hybrid electronic medical record system for the surveillance of adverse surgical events and human error in a developing world surgical service. World J Surg 2015;39(1):70-79. DOI:10.1007/s00268-014-2766-x

5. Laing GL, Bruce JL, Clarke DL. Tick-box admission forms improve the quality of documentation of surgical emergencies but have limited impact on clinical behaviour. S Afr Med J 2014;104(6):435-438. surgical emergencies but

6. Vinen J. Incident monitoring in emergency departments: An Australian model. Acad Emerg Med 2000;7(11):1290-1297.

2000;7(11):1290-1297.
Stewart WWD, Farina Z, Clarke DL, Thomson SR. Variations in levels of care within a hospital 7. Stewart WWD, Farina Z, Clarke DL, Thomson SR. Variations in
provided to acute trauma patients. S Afr J Surg 2011;49(4):194-198.

8. Clarke DL, Furlong H, Laing GL, Aldous C, Thomson SR. Using a structured morbidity and mortality meeting to understand the contribution of human error to adverse surgical events in a South African regional hospital. S Afr J Surg 2013;51(4):122-126. DOI:10.7196/SAJS.1537

9. Levtzion-Korach $\mathrm{O}$, Frankel A, Alcalai $\mathrm{H}$, et al. Integrating incident data from five reporting systems to assess patient safety: Making sense of the elephant. Jt Comm J Qual Patient Saf 2010;36(9):402-410.

10. Christians-Dingelhoff I, Smits M, Zwaan L, Lubberding S, van der Wal G, Wagner C. To what extent are adverse events found in patient records reported by patients and healthcare professionals via complaints, claims and incident reports? BMC Health Serv Res 2011;11:49. DOI:10.1186/1472-6963$11-49$

11. De Feijter JM, de Grave WS, Muijtjens AM, Scherpbier AJ, Koopmans RP. A comprehensive overview of medical error in hospitals using incident-reporting systems, patient complaints and chart review of of medical error in hospitals using incident-reporting systems, patient complaints and
inpatients deaths. PLoS One 2012;7(2):e31125. DOI:10.1371/journal.pone-0031125

12. Clarke DL, Gouveia J, Thomson SR, Muckart DJ. Applying modern error theory to the problem of . Clarke DL, Gouveia J, Thomson SR, Muckart DJ. Applying modern error theory to the proble
missed injuries in trauma. World J Surg 2008;32(6):1176-1182. DOI:10.1007/s00268-008-9543-7 missed injuries in trauma. World J Surg 2008;32(6):1176-1182. DOI:10.1007/s00268-008-9543-7
3. Nam J. Comparison of validity of assessment methods using indices of adjusted agreement. Stat Med 2007;26(3):620-632. DOI:10.1002/sim.2562

Accepted 7 February 2016. 


\section{Appendix 1. RF1 form: Adverse event detection questionnaire}

\section{CONFIDENTIAL}

\section{RF1: adverse event detection questionnaire}

\section{REVIEWER}

Reviewer ID Number:

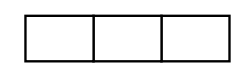

Clinical department $\mathrm{n}^{\circ}$

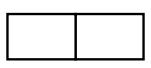

Date of data collection:

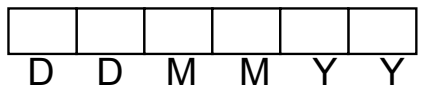

Time interview commenced: (use 24 hour clock)

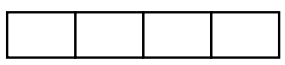

Time interview finished:

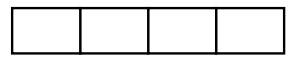

PATIENT NAME:

(Surname)

\section{CASE Number}

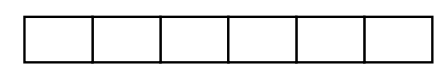

Birth date (at least the year of birth)

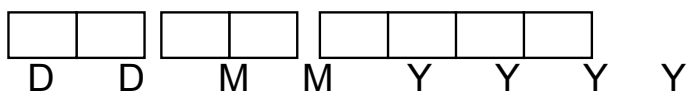

Gender (1 male / 2 female)

Admission Status

( 1 elective / 2 acute / 3 do not know)

Date of Admission:

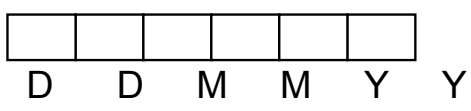

Date of Discharge (if known):

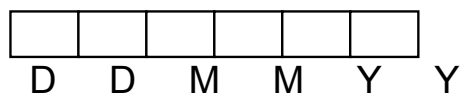




\title{
SCREENING CRITERIA
}

1. Unplanned admission in the 12 months prior to the index

$$
\square 1=\text { Yes } 2=\text { No }
$$

\begin{abstract}
2. Hospital-incurred patient accident or injury.
\end{abstract}


3. Adverse drug reaction / drug error

$\square 1=$ Yes $2=$ No

4. Hospital acquired infection/sepsis.

$1=$ Yes $2=$ No

5. Unplanned removal, injury or repair of organ or structure during surgery, invasive procedure or vaginal delivery.

6A. Unplanned return to the operating theatre during this admission $\square 1=$ Yes $2=$ No

B. Unplanned visit to the operating theatre during this admission 
7. Unplanned open surgery following closed or laparoscopic surgery.

8. Cardiac/respiratory arrest, low apgar score.

9. Development of neurological deficit not present on admission

$\square=$ Yes $2=$ No

10. Injury or complications related to termination of pregnancy or labour and delivery including neonatal complications.

11. Other patient complications to include: MI, DVT, CVA, PE etc. $\square 1=$ Yes $2=$ No

12. Patient/family dissatisfaction with care received documented in the medical record and/or documentation of claim or litigation 
13. Unplanned transfer from general care to intensive care/ higher dependency.

14. Unplanned transfer to another acute care hospital $1=$ Yes $2=$ No

15. Unexpected death (i.e. not an expected outcome of the disease during hospitalisation)

16. Any other undesirable outcomes (not covered by any $1=$ Yes $2=$ No of the other criteria).

Are any Criteria present?
$1=$ Yes

$2=$ No $($ Then STOP; do not the second questionnaire)

If Yes, total number of criteria 


\section{Appendix 2. RF2 form: Second questionnaire}

\section{Second questionnaire (RF2)}

\section{Take account of all adverse events identified in RF1, not only the most serious. Complete one form for each adverse event(AE) \\ If the patient has more than one AE, use two RF2 questionnaires and quote "1 out of 2" and "2 out of 2"}

Case number (same number as in RF1).

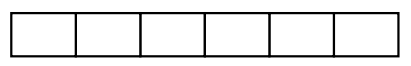

Reviewer ID Number:

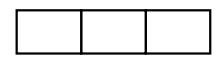

Date of data collection:

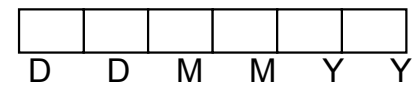

Time when interview commenced:

(use 24 hour clock)

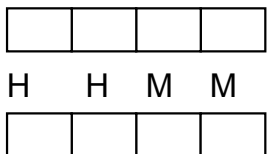

Time interview finished:

\begin{tabular}{|l|l|l|l|}
$\mathrm{H}$ & $\mathrm{H}$ & $\mathrm{M}$ & $\mathrm{M}$ \\
\hline & & & \\
\hline
\end{tabular}

Q1 \& Q2 AE No $\square$ out of a total of $\square$ AE

Information sources used

Q3 Physician

$\quad I=$ Yes $2=$ No

Q4 Head Nurse

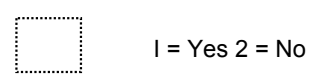

Q5 Nurse

$$
\quad \mathrm{I}=\text { Yes } 2=\text { No }
$$

Q6 Medical record

$\mathrm{I}=$ Yes $2=$ No

Q7 Other source

$I=$ Yes $2=$ No 
Q8 Did the patient experience an adverse event (injuries or complications)?

Q9 When did this event occur?

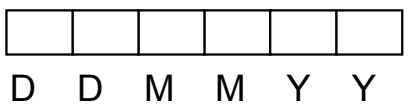

Q10 Clinical summary of the case and description of the adverse event Main disease

Known comorbidities

History of disease (in particular specify if the disease was known before admission)

Cause for hospital admission

Main events during hospitalisation

Adverse event: (for example, answer briefly the following "what, who, when, where, how" questions) (Give any relevant laboratory/imaging results) continue on back if needed 


\section{ADVERSE EVENT DETERMINATION}

SEVERITY (answer : $1=$ Yes $2=$ No)

Q11 Did the injury or complication caused the hospitalisation?

Q12 Was the injury or complication associated with death of the patient

Q13 Was the injury or complication associated with disability/deficit at the time of discharge?

Q14 Was the injury or complication associated with prolonged hospital stay? (including readmission)

\section{CAUSATION}

Q15 In your best judgement, is there evidence that healthcare management caused the adverse event? In answering this question, consider, when relevant, the following questions and complete the appropriate boxes.

Q151 Could the event be expected, giving the disease or the health status of the patient? $1=$ Yes 2 = No 3 = Don't know

Q152 Are there indications that health care management caused the injury?

$1=$ Yes 2 = No 3 = Don't know

Q153 Does the timing of events suggest that the injury was related to the treatment or lack of treatment? $1=$ Yes $2=$ No $3=$ Don't know

Q154 Are there other reasonable explanations for the event? I = Yes 2 = No 3 = Don't know
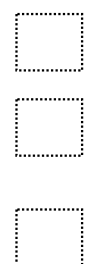

Q155 Is there general recognition that the intervention or lack/delay of intervention or diagnosis (depending in the instance) causes this kind of adverse event? $1=$ Widely recognised by scientific community $2=$ Recognised by some specialists only $3=$ No recognition $4=$ Don't know

Q156 Was there an opportunity prior to the occurrence of the injury for intervention which might have prevented it? $\mathrm{I}=$ Yes $2=$ No $3=$ Don't know

Q157 Was the AE recognised during the hospital stay? $1=$ Yes $2=$ No

Q157a Was appropriate action taken? 1= Yes 2= No 3 = not applicable

Q157b Did the AE respond to the appropriate action? $1=$ Yes $2=$ probably $3=$ too early to know 4 No 5 Don't know 6 Not applicable

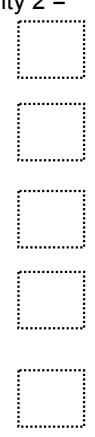

\section{Consider all of the above questions above before continuing}

Q16 After due consideration of the clinical details of the patient's management, irrespective of preventability what level of confidence do you have that the HEALTH CARE MANAGEMENT caused the injury?

\section{Confidence Score:}

1 = Virtually no evidence for management causation (Then STOP, no AE)

2 = Slight to modest evidence for management causation (Then STOP, no AE)

3 = Management causation not likely; less than 50-50 (Then STOP, no AE)

4 = Management causation more likely than not, more than 50-50

$5=$ Moderate/strong evidence for management causation

6 = Virtually certain evidence for management causation

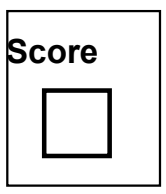

The questionnaire is complete if your score is three or less 


\section{Q17 Location of occurrence}

Q171 Where did the healthcare management causing the AE occur? (choose one) 1 outside this hospital 2 inside this hospital

\section{Q172 If outside this hospital}

$01=$ Public hospital

$02=$ Private hospital

$03=$ Home with professional healthcare management

$04=$ Home without professional healthcare management

$05=$ Nursing home

$06=\mathrm{GP}$ office

$07=$ other

Q173 If inside hospital, in the clinical unit in which the patient was hospitalised?? Yes $2=$ No

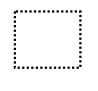

\section{Q174 If not in patient's clinical unit, in which one?}

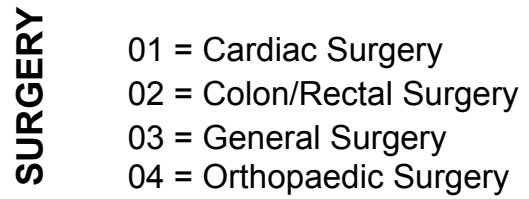

$$
\begin{aligned}
& 05=\text { Paediatric Surgery } \\
& 06=\text { Plastic Surgery } \\
& 07=\text { Thoracic Surgery } \\
& 08=\text { Urological Surgery } \\
& 09=\text { Vascular Surgery }
\end{aligned}
$$
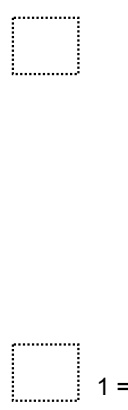

$15=$ Cardiology

$16=$ Dermatology

Ш $17=$ Endocrinology

$18=$ Gastroenterology

$19=$ Geriatrics

$20=$ Gynaecology

$21=$ Haematology

22 = Immunology and Allergy
$23=$ Infectious Disease

24 = Internal Medicine

(not otherwise classified)

$25=$ Physical Medicine

$26=$ Neonatology

$27=$ Nephrology

$28=$ Neurology

29 = Medical Oncology

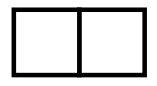

$10=$ Neurosurgery

$11=$ Obstetrics

$12=$ Ophthalmology

$13=$ ENT

$14=$ Stomatology

Q18 If inside hospital, where exactly?

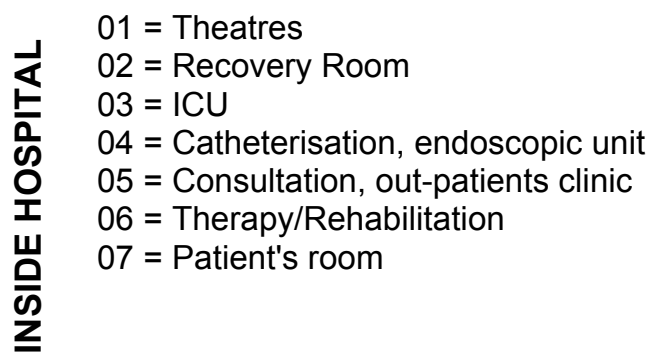

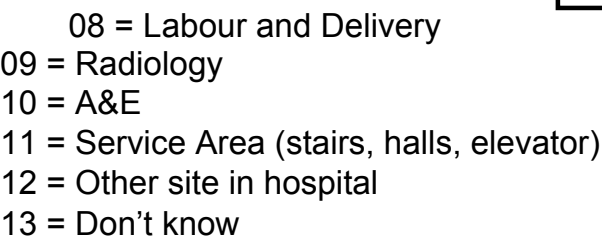

$30=$ Paediatrics

31 = Pulmonary Disease

32 = Psychiatry

$33=$ Medical Intensive Care Unit

34 = Rheumatology

$35=\mathrm{A} \& \mathrm{E}$

$36=$ Other 


\section{Q19 CLASSIFICATION OF ADVERSE EVENT}

Q191 To which type of care management was the adverse event mainly related? 1 prevention 2 diagnosis 3 therapeutic 4 rehabilitation

Q192 What was the main cause of $A E$ (the most important one)

$1=$ Error in the choice of management

2 = Delay for its implementation

$3=$ Error during its implementation.

$4=$ Other (mainly unavoidable events)

$5=$ Don't know

Q193 Was the AE related to a procedure? ? $1=$ Yes $2=$ No

Q193a If yes, which procedure?

1 = Surgery

2 = Anesthesiology

3 = Surgical intervention during radiology.

4 = Radiology using contrast product

5 = Endoscopy

6 = Biopsy

$7=$ Puncture or tapping

$8=$ Catheter, perfusion or injection

$9=$ Urinary catheter

$10=$ Gastric

$11=$ Intubation

$12=$ Dialysis

$13=$ Radiotherapy

$14=$ Instrument assisted delivery

$15=$ Physiotherapy

$16=$ Other

Q194 Was the AE related to a substance or health product? ? $1=$ Yes $2=$ No

Q194a If yes, which product?

$1=$ drug

2 = blood product

$3=$ medical device.

4 = equipment (laser, electric bistoury..)

$5=$ Dietetic product

6 = Local preparation (e.g. chemotherapy product...)

$7=$ Other

\section{Q20 Patient-related contributory factors?}

Q201 Patient's global health status and disease

Q202 Patient's behaviour

$1=$ Yes $2=$ No

Q203 Family's behaviour

$1=$ Yes $2=$ No

Q204 Other

Specify:

$1=$ Yes $2=$ No

$1=$ Yes $2=$ No

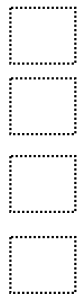




\section{Q21 System-related contributory factors? $1=$ Yes $2=$ No}

(For all 16 next items, answers are $1=$ Yes $2=$ No)

Q211 Inadequate or defective premises

Q212 equipment or supplies not available or defective

Q213 inadequate staffing at the time of the $A E$ (not merely in terms of numbers, take account of balance among differe........ competences and experience, in particular at weekend and during holidays))

Q214 recent organizational changes inside the unit

Q215 defective coordination inside the unit

Q216 inadequate reporting or communication

Q217 inadequate training or supervision of doctors or other personnel

Q218 delay in the provision or scheduling of services (e.g. lab tests, x-rays or follow-up visits)

Q219 failure to implement protocol or plan

Q220 inadequate monitoring of patient

Q221 inadequate discharge procedure

Q222 defective coordination between the unit and other units (e.g. pharmacy, blood bank or catering)

Q223 No protocol/healthcare policy

Q224 Other

Describe the most important contributing factor to the adverse event

Q23 inappropriate adaptation to an unexpected event

Describe inappropriate adaptation 


\section{Q24 PREVENTABILITY}

Consider and evaluate the following questions before making a judgement on preventability.

Q241 How serious was this case PREVIOUS to the occurrence of an AE?

$1=$ Very serious

$2=$ Moderately serious

$3=$ not very serious

$4=$ Not serious

Q242 How complex was this case? (co-morbidity, global health status)

$1=$ Very complex

$3=$ not very complex

$2=$ Moderately complex

$4=$ Uncomplicated

Q243 What was the degree of emergency in management of the case prior to the occurrence of adverse event?

$1=$ Critical and very urgent $\quad 3=$ low

$2=$ Moderate $\quad 4=$ Not urgent

Q244 Was the management of the illness appropriate?

$1=$ Large consensus

$3=$ No consensus

$2=$ Consensus moderate

$4=$ Management non-indicated or contra-indicated

5 = Don't know

Q245 What was the degree of deviation of management from recommendations?

$1=$ None

2 = Slight

$$
\begin{aligned}
& 3=\text { Moderate } \\
& 4=\text { Marked } \\
& 5=\text { Don't know }
\end{aligned}
$$
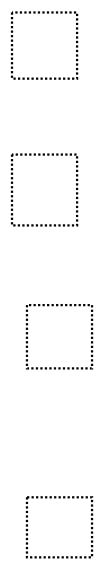

Q246 What was the chance of benefit associated with the management of the illness which led to the AE?

$$
\begin{array}{ll}
1=\text { High } & 3=\text { Low } \\
2=\text { Moderate } & 4=\text { absent }
\end{array}
$$

Q247 What was the risk of an adverse event related to the management?

$$
\begin{aligned}
& 1=\text { virtually absent } \quad \begin{array}{l}
3=\text { Moderate } \\
2=\text { low }
\end{array} 4=\text { High }
\end{aligned}
$$

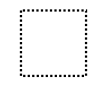

Q248 On reflection, would a reasonable doctor or health professional have managed the care in a similar manner?

$1=$ Definitely would 2 = Probably would

$$
\begin{aligned}
& 3=\text { Probably would not } \\
& 4 \text { = Definitely would not }
\end{aligned}
$$


Consider all the questions 241-248 above before continuing

25 Rate on a 6 point scale your confidence in the evidence for preventability.

Confidence score:

$1=$ Virtually no evidence for preventability

2 = Slight to modest evidence for preventability

$3=$ Preventability not really likely; less than $50-50$

$4=$ Preventability more likely than not; more than $50-50$

$5=$ Strong evidence for preventability

$6=$ Virtually certain evidence for preventability

26 Please describe in which way the adverse event may have been prevented 